TITLE: PARALLEL GRAPH REDUCTION ON A SUPERCOMPUTER:

\title{
A STATUS REPORT
}

\author{
AUTHOR(S): Randy Michelsen, C-10 \\ Lauren Smith, C-8 \\ Elizabeth Williars, C-8 \\ Bonnte Yantis, C-10
}

Suemitted to: To appear in 'Proceedings of Santa Fe Graph Recuction Workshop." Springer-Verlag

\section{DISCLAIMER}

This report was g.repared as un account of work sponsored by an agency of the United Siates (iovernment. Neithef the United States (iovernment nor uny agency thereof, nor uny of their employees, makes uny warranty, express or implied, or usumes any legal liability or renponsihility for the uccuracy, completeness, or usefulness of uny informution, uppuratus, product, or process disclosed, or represents that its use would not infringe privately owned rights Reference herein to any specific commercial producl, process, or service hy trnde name. trademark. manufucturer, of otherwise does not necessarily conatitute or imply its endorsement, recommendation, or favoring by the Uniled Stales Ciovernment or any agency thereor. The views und opinions of authors expressed hersin do not necessarily state or reflect thone of the United Stalen (iovernment or any agency thereol. 


\title{
Parallel Graph Reduction on a Supercomputer: A Status Report
}

\author{
Randy Michelsen \\ Lauren Smith ${ }^{1}$ \\ Elizabeth Williams \\ Bonnie Yantis \\ Computing and Communications Division ${ }^{2}$ \\ Los Alamos National Laboratory \\ Los Alamos, NM 87545
}

\begin{abstract}
We describe an ongoing effort to develop a parallel graph reduction run-time system hosted on a multiprocessor supercomputer. This run-:ime syste:m is presently augmented by the functional language comoiler of the ALFALFA system. Admittedly, parallel graph reduction is hardly a novel idea. The interesting notion is the provision of a parallel execution environment sufficiently powerful to support development of large prototypical scientific and symbolic codes in a functional language. This will allow the investigation of the functional programming paradigm within these application domains in an empirical fashion. In this paper, we discuss the motivation for the effort, describe the basic elements of the implementation, and provide some preliminary insight distilled from our experience with an initial version of the run-time system.
\end{abstract}

\section{INTRODUCTION}

The continuing demand for increased computational power, especially in the realm of scientific computing, has spurred interest in parallel compulation. Today, most application development for computationally intensive algorithms intended for high-performance computers is done in Fortrnn. Hence, there exists a considerable bocy of knowledge regarding its use and performance in such domains. We are interested in collecting similar data relating to use of the functional (or applicative) paradigm.

Toward this end, we are currently developing a parallel graph reduction run-time system for a supercomputer incorporating four high-speed processors. The code generation is perfoimed by an existing functional language compiler resident on a traditional uniprocessor. Initially, the focus of the effort was the conversion, with minimal change, of a run-time system originally developed for a distributed memory multiprocessor to a shared memory muitiprocessor. This strategy enabled is, in the near term, to rely upon an existing compiler and concentrate our energy instead on the development of the reduction run-time system. Subsequently, the emphasis will shift to performance tuning of the run-time system arid the investigation of related compilation and scheduling issues.

Our motivation for undertaking this :ask was essentially twofold. A common complaint from potential users of functional languages outside of the language re:search community is the pragmatic impossibility of developing programs of a "realistic" size simply due in performance-related issues. The definition of realistic is of course open to interpretation, but it is 'worthy of note that lypical production programs at Los Alamos National I aboratory (hereafter, the l aboratory) consist of $((X),(X) X)$

1 currently at Department of Defense, 9800) Savage Road, Fon Meade, MD 20755, elexirunic: mail iddress: Ils(a)mimsy.umd.edu

2 This work was perforned iniler the auspices of the Department of linerky. 
or more lines of Fortran. The purformance deficiencies usually associated with functional languages may be attributed in part to the fact that most implementations currently available are intended for language research and lack the maturity (and implied fine tuning) of production compilers. Furthermore, the implementations generally exist on machines lacking the requisite processing and memory capacity for large-scale scientific computations. Our effort is particularly apropos given thi latter circumstance.

We were also interested in exploring the performance ramifications of a large physical memory shared by cooperating reduction "engines." There have been graph reduction systems implemented on distributed memory machines or networks of machines, including several discussed in this volume. Yet, given the usual logical view of a graph space global to all reduction activity, a large shared memory architecture seems particularly well suited to parallel graph reduction.

As a result of these interests and practical concerns such as machine availability, we chose the CRAY X-MP computer as the current implementation target. This architecture combines a small number of high-performance processors with a large shared memory.

Section 2 contains background information and a brief discussion of the CRAY X-MP architecture. [2] Section 3 describes the design of the initial run-time system. Section 4 discusses early experience with the system and a recently initiated follow-up development effort. A summary is presented in Section 5.

\section{BACKGROUND}

\subsection{The ALFALFA System}

The work described here is closely related to the work on ALFALFA by Benjamin Goldberg and Paul Hudak of Yale University. We shall present a very brief description of the ALFALFA system, but the interested reader is referred to B. Goldberg's paper in this volume for a more complete treatment of the subject. ALFALFA consists of a compiler for the functional language ALFL [3] and an associated parallel graph reduction system resident on an Intel iPSC multiprocessor. A source program is parsed into an intermediate graph form called LIF (Lambda Intermediate Form). This graph is then annotated as a result of extensive type, sharing, and strictness analysis. The annotated graph is converted into a serial combinator [ 41 prograin graph. Finally, $C$ procedures are generated from this graph for the combinators. This $\mathrm{C}$ program is then compiled and linked with the graph reduction system resident on tl:e processing nodes of iPSC.

\subsection{The CRAY X-MP}

The CRAY X-MP is a multiprocessor derivative of the earlicr CRAY-1 family of supercomputers. The basic architeclure consists of an interleaved, multiport memory and input/output subsystems shared by several identical high-performance vector processors. Each processor has a major cycle time of $\mathbf{8 . 5}$ nanoseconds. Pipe-lining is utilized in the scalar and vector functional units, with parallel activity possible among the independenc functional uuits. A parallel program has available to it shared registers - eight data registers, eight address registers, and 32 single-bit semaphore registers. A blocking test-and-set instruction can be performed on each semaphore register. Unlike some of its counterparts, the X-MP does not support virtual memory, but rather uses a contiguous allocation scheme for memory management. The $\mathrm{X}-\mathrm{MP}$ is available in a variety of configurations, currenily with up to four processors and sixteen million 64-bit words of main memory.

The primary use of modem supercomputers as "number crunchers" is retlected in the software environment of the Cray. The most malure and heavily used compiler available at the laboratory is the vectorizing Fortran compiler developed by ('ray Resedreh Ine. liortunately, a lexally supported ( compiler is also available. The C language wati clearly more altractive in our development beciase of portability concerns and the availubility of languige leatures such as dynamically allex:attihle datti strictures. 
The processors of a CRAY X-MP have traditionally been used to support multiple, indeps:ndent job streams. However, a set of routines has been developed at the Laboratory to enable user program iniciation and control of parallel tasks. These multitasking routines [1], written in Fortran and Cray assembly language, provide constructs for task creation, termination, and various styles of cooper:tion among asynchronous tasks. Among the latter are analogues of traditional syachronization mechanisms such as generalized counting semaphores, fetch/add instructions, locks, event;, barriers. forks, and joins. Because of the real memory organization of the X-MP, the multitasking routines rely upon memory management utilities developed and in use at the Laboratory.

\section{THE CRAY GRAPH REDUCTION RUN-TIME SYSTEM}

Since one of our intentions was to provide access to Cray-class computational power for functional language programs in a timely fashion, the initial version of the Cray reduction system is a variant of the ALFALFA system developed for the Intel iPSC. Of course, given the disparate target architectures of the two efforts, there are considerable differences to accommodate. We are currently using the compiler from the ALFALFA system as the code generator for this reduction system. The $C$ source code produced by the compiler undergoes source-to-source transformations to produce the appropriate Cray-compatible code. At this point, the source code is loaded onto the Cray. These combinator definitions are compiled and linked with the resident run-time system.

An alternative design for the run-time system was considered, but eventually rejected in favor of the design presented in this section. A primary goal in this initial implementation was tc produce a run-time system highly compatible with the original ALi'ALFA compilation system, with sorie consideration given to performance factors. The candidate design was to associate with each node being reduced a separate reduction run-time system, with scheduling of these tasks performed by the host's operating system. This design was rejected because of the high overhead of task management relative to the actual work associated with the reduction activity.

In the selected design (refer to Figure 1), each physical processor executes a reduction task that is the run-time system. The term "task" is used in this context to designate an executing program: image on the Cray host. The program graph and a queue of ready nodes, i.e., graph nodes that are candidates for immediate reduction, reside in shared memory. The common node queue is accessed by the reduction tasks whenever new work is desired. Access to this queue and the nodes of the program graph is controlled through the use of synchronization variables. Error handling and normal termination of the run-time system are accomplished via the notion of "synchronized meetings" provided by the multitasking routines. This construct allows a set of parallel tasks to synchronize on a single variable, denoted us the "meeting flag." After all the tasks meet at this synchronization point, a single task executes a sequentia' section of code. Upon the completion of this ection of sode, all tasks resume parallel execution. A varian! of this design uses a fixed number of reduction tasks, which is strictly greater than the number of processors. When one reduction task must wait to gain access to a lock. another task may be able to reduce a node if one is on the ready queue: thus. all processors are executing if nodes are ready.

As indicated earlier, the run-time system is written primarily in C. Because of the n:ed to interface the Fortran multitasking routines and the iun-time system, a Fortran main program actually initiates the bootstrap routine for the run-time system. The $\mathrm{C}$ bootstrap routine initializes the ready node queue, the various synchronization variables, and constructs the inirial program graph. When the in:tialization is complete, four parallel reduction tasks are created. One of the reduction tasks determines that it is the sont (using a shared variable) and initiates the graph reduction. "The tasks continuc sclecting nodes from the ready queue, mutating the program graph, and placing nodes on the ready queue until the gruph is reduced to normal form or there is an abnorme! termination.

$I /()$, because of its sequential nature, is accomplished through the use of a sofiware "lock" ill the $1 /()$ module. When one of the reduction tasks needs to perform $1 / 0$, it altempts lo gatin pessiession of the I/() lock. If the lox.k is unavailable (i.e., already mossessed by another task), the requesting talsk waits until if is available. Mernory allexation and deallexation are handled in a similar fishion.

lirror handling is accomplished by a synchronized meeting. If a serious error is detected by one of the reduction tasks, the task sets the synchronization variable and initiates a meeling. The ofler 


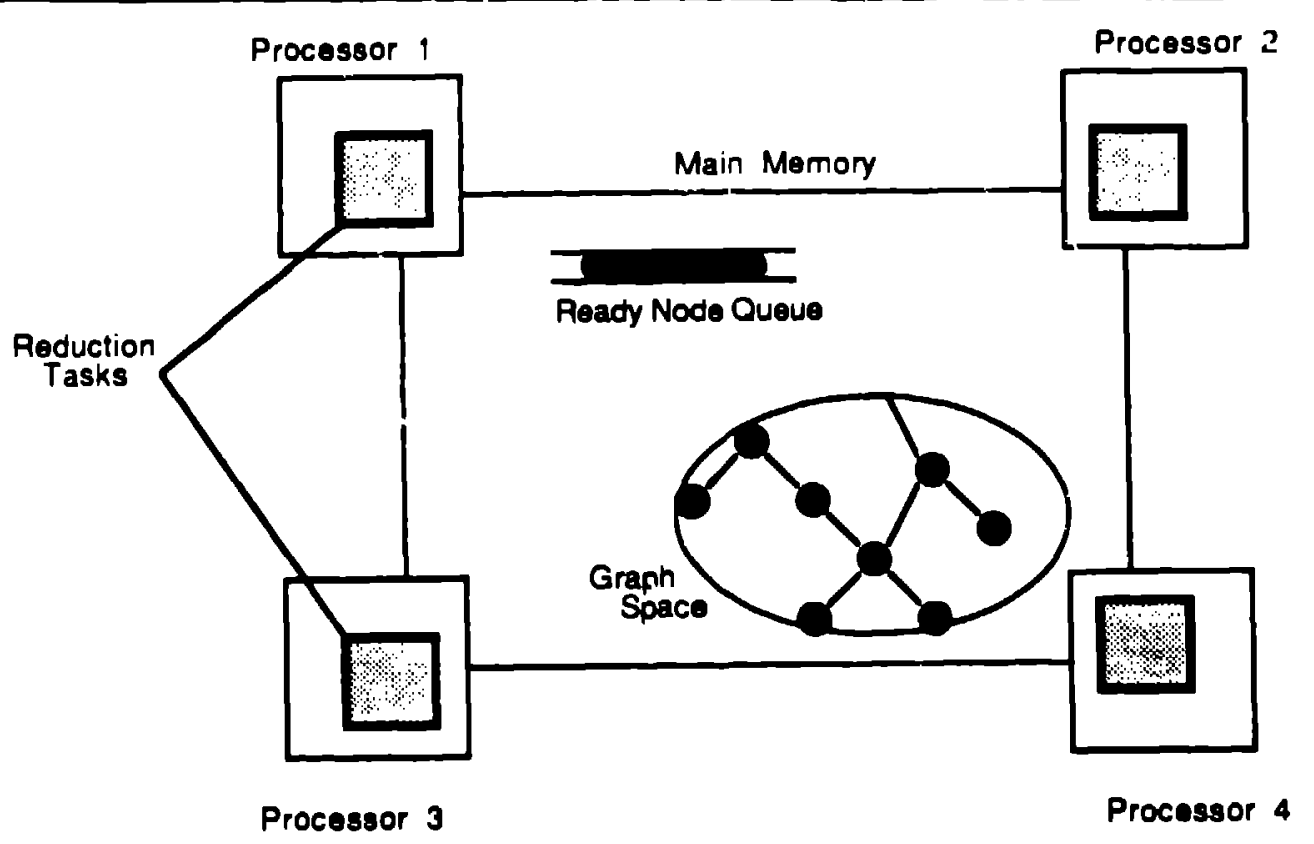

Figure 1. The Cray run-time system.

reducers will synchronize and the initiatiry task will execute the appropriate error handling. To ensu:e correct behavior in the advent of a meeting requested as a result of a fatal error, the tasks of the run-time system periodically inspect the meeting flags associated with this event. This inspection is critical in order to avoid potential deadlock as a result of tasks waiting for locks retained by abnormally terminated tasks. The occurrence of a fatal error will sause logging of state information, foilowed by the termination of the reduction tasks.

\section{F.ARLY EXPERIENCE.}

At this point, the reduction system has been applied to simple ALFL programs. Several performance obstacles have been identified on the hasis of our limited experience with the Cray environment, including the multitasking routines. The granu'arity of computation is far too small to be practical given the raw performance characteristics of the Cray processor. For example, the overhead associated with a procedure call is approximately $\mathbf{4 0}$ major cycles. This indicates a need to increase the granularity of the combinators generated by the compiler, as well as a need to significantly restructure the reduction system itself to reduce the cumulative performance impact of procedure invocation. Another obvious candidate for further sciutiny is synchronization. The tactic in this instance must be to reduce points of synchronization and to minimize the overhead associated with each synchronization.

These issues, among others, have been addressed $\mathrm{i}$ :, a subsequent development project targeted toward enhanced performance. In particular, the reduction system was redesigned to benefit more fully from the shared memory model, to more accurately ieflect the performance characteristics of the Cray architecture, and to support measurement of performance and workload characteristics. Through careful design and utilization of a different mechanism, the minimum overhead incurred io control access to shared objects such as the ready node queue has been reduced by a factor of 7 io 1.5 . linally, an alternative scheme for internal data representation has been emplnyed that is more amenable to possible future optimizations. 'This scheme supports memory allox'ation in a mather such that effective utilization of the Cray vector hardware is pessible where appropriate. 


\section{SUMMARY}

We briefly' discussed the status of a parallel graph reduction system for the CRAY X-MP multiprocessor. A :arge portion of the initial effort was devoted to, out of sheer necessity, deciphering the interactions of several disparate sofiware systems (e.g., memory management utilities). This effort will be fully appieciated by those acquainted with the rather meager software development support tools traditionaliy available on supercomputers.

The poor performance of the initial version of the run-time system prompted the development of a second redesigned system. This system is presently undergoing integration testing. This version has been tailored for increased performance on the host architecture. In particular, synchronization and other operational overheads have been significantly reduced.

In the future, we anticipate performing characterization studies of prototypical applications and additional performance tuning of the run-time system. The former should provide important data for the investigation of issues such as task granularity and scheduling. Finally, the entire question of vectorization has been ignored but must be addressed in any truly viable high-performance system.

\section{REFERENCES}

(1) Bobrowicz, F., "The Los Alamos Multitasking Control Library," Los Alamos National Laboratory unclassified release (in preparation).

(2) Cray Research, Inc., CRAY X-MP Series Mainframe Reference Manual, HR-0032 (1982), Cray Research, Inc.

(3) Hudik, P., "ALFL Reference Manual and Programmer's Guide." Research report YALJ:U/DCS/RR-322, 2nd ed., Computer Science Departnent, Yale University, October 1984.

(4) Hudak, P., and Goldberg, B., "Distributed execution of funitional programs using serial combinators," finr. 1985 International Conference on Parallel Processing, August 1985, pp. 831 839. 